\title{
Remodeling immune microenvironment in periodontitis using resveratrol liposomes as an antibiotic-free therapeutic strategy
}

Junyu Shi ${ }^{1 \dagger}$, Yi Zhang ${ }^{1,2 \dagger}$, Xiaomeng Zhang ${ }^{1}$, Ruiying Chen ${ }^{1}$, Jianxu Wei ${ }^{1}$, Jiazhen Hou $^{2}$, Bing Wang ${ }^{2}$, Hongchang Lai ${ }^{*}$ and Yongzhuo Huang ${ }^{2,3,4,5^{*}}$

\begin{abstract}
Background: Periodontitis is a complicated inflammatory disease that damages the tooth-supporting tissues, with limited pharmacotherapy available. Macrophage-targeting therapy is promising for inflammatory diseases. Resveratrol (RSV), a nonflavonoid polyphenol, is known for its anti-inflammatory and immunomodulatory effects. However, its medical application is limited by its poor stability and water-solubility, as well as its low bioavailability.

Result: A therapeutic resveratrol-loaded liposomal system (Lipo-RSV) was developed to treat periodontitis. The physical properties of Lipo-RSV and its ability to regulate macrophages were investigated. The results showed that Lipo-RSV had good biocompatibility and could re-educate the inflammatory macrophages from M1- to M2-like phenotype through activating p-STAT3 and downregulating p-STAT1. Besides, the Lipo-RSV could scavenge ROS and inhibit the NF-KB signal and inflammasomes, thereby reducing the pro-inflammatory cytokines IL-1 $\beta, I L-6$, and TNF-a.
\end{abstract}

Conclusion: These results revealed that Lipo-RSV could be a potential therapeutic system for the antibiotic-free treatment for periodontal diseases.

Keywords: Resveratrol, Macrophage, Periodontitis, Liposome, Immune microenvironment, Local delivery

\footnotetext{
*Correspondence: laihongchang@sjtu.edu.cn; yzhuang@simm.ac.cn

†Junyu Shi and Yi Zhang Contributed equally to this work

${ }^{1}$ Department of Implant Dentistry, Shanghai Ninth People's Hospital,

Shanghai Jiao Tong University School of MedicineCollege of Stomatology,

Shanghai Jiao Tong University; National Center for Stomatology, National

Clinical Research Center for Oral Diseases; Shanghai Key Laboratory

of Stomatology, 639 Zhizaoju Road, Shanghai 200011, China

${ }^{2}$ State Key Laboratory of Drug Research, Shanghai Institute of Materia

Medica, Chinese Academy of Sciences, 501 Haike Rd, Shanghai 201203,

China

Full list of author information is available at the end of the article
}

(c) The Author(s) 2021. Open Access This article is licensed under a Creative Commons Attribution 4.0 International License, which permits use, sharing, adaptation, distribution and reproduction in any medium or format, as long as you give appropriate credit to the original author(s) and the source, provide a link to the Creative Commons licence, and indicate if changes were made. The images or other third party material in this article are included in the article's Creative Commons licence, unless indicated otherwise in a credit line to the material. If material is not included in the article's Creative Commons licence and your intended use is not permitted by statutory regulation or exceeds the permitted use, you will need to obtain permission directly from the copyright holder. To view a copy of this licence, visit http://creativecommons.org/licenses/by/4.0/. The Creative Commons Public Domain Dedication waiver (http://creativeco mmons.org/publicdomain/zero/1.0/) applies to the data made available in this article, unless otherwise stated in a credit line to the data. 


\section{Graphical Abstract}

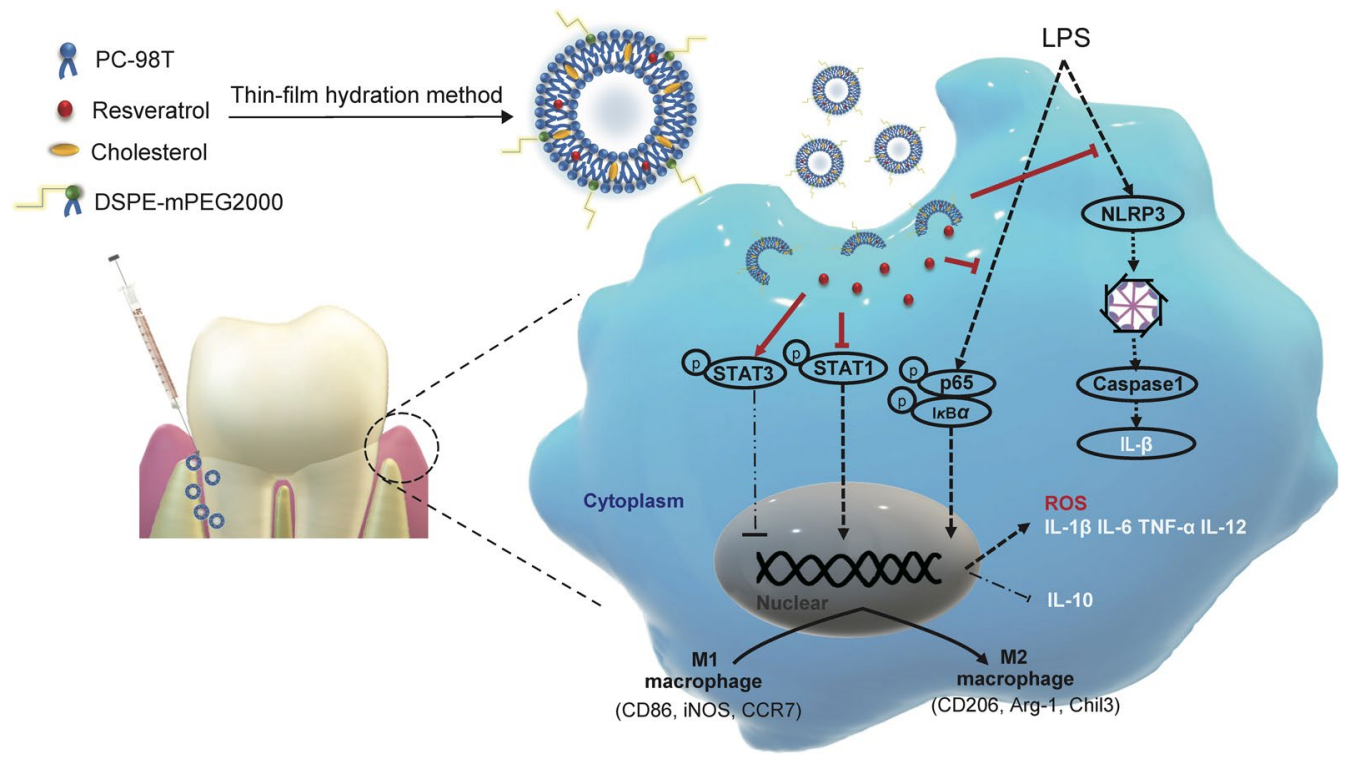

\section{Introduction}

As one of the most prevalent oral diseases, periodontitis can affect up to $90 \%$ of the global population, and it has been identified as a leading cause of tooth loss in adults [1]. Periodontal pockets act as bacterial niches and provide favorable conditions for microbial proliferation. Periodontitis also raises the risk of systemic diseases such as cardiovascular diseases, Alzheimer's disease, and inflammatory bowel diseases [2]. Mechanical debridement and adjunctive antibiotics remain the mainstay of treatment. However, the treatment outcomes are often compromised by the development of antibiotic resistance and drug interactions [3]. Also, the heavy use of antibiotics could further exacerbate the global crisis of antibiotic abuse [4]. Therefore, it is urgent to find new alternative treatment strategies for periodontitis [5].

Although plaque is considered to be the initiating factor in periodontitis, it has been well accepted that immune disorders further worsen the situation and non-resolving inflammation is consequently developed, which is the main cause of periodontitis-related tissue damage [3, 6]. Therefore, the traditional single antibacterial therapy may not be sufficient to solve such a complex problem of periodontitis [7]. Targeting the immune microenvironment could be a promising strategy for periodontitis [8].

Macrophages, a main regulator of the innate immune system, play a key role in controlling the inflammation process [9]. It has been revealed that targeting macrophages is a potential therapeutic intervention for periodontitis $[10,11]$. For example, the repolarization of macrophages from M1-like inflammatory phenotype to M2-like anti-inflammatory phenotype can effectively ameliorate periodontitis [8].

Resveratrol (RSV) is a natural nonflavonoid polyphenol derived from several plant species and has anti-inflammatory and antioxidant effects [12]. For instance, resveratrol could regulate macrophage polarization to inhibit the inflammatory mediators in myocardial infarction [13] and acute gouty arthritis [14]. However, the therapeutic use of resveratrol is restricted due to its poor stability and water-solubility as well as the low bioavailability [15]. Therefore, an effective delivery system of resveratrol is important for its clinical application.

Nano-drug delivery systems can be used to improve the efficacy of drugs on macrophage reprogramming [16]. In this work, a macrophage-repolarization nanotherapeutic strategy was investigated for remodeling the immune microenvironment of periodontitis. The resveratrol liposomes were developed for local administration to the periodontal pocket to treat periodontitis. Liposomal formulations are among the most druggable nanocarriers, and thus naturally considered a priority in the study due to the translation potential.

\section{Materials and methods \\ Materials, cells, and animals}

Resveratrol and ibuprofen was purchased from MeilunBio (Dalian, China). Minocycline Hydrochloride was purchased from Sunstar Co., Ltd (Takatsuki, Japan). Dulbecco's modified Eagle's Medium (DMEM) and fetal 
bovine serum $\left(\mathrm{Gibco}^{\circledR}\right)$ were obtained from Thermo Fisher (Waltham, USA). The cocktail protease inhibitor, lipopolysaccharide, and Pluronic ${ }^{\circledR}$ F127 were purchased from Sigma-Aldrich (St. Louis, USA). P.g-LPS was from InvivoGen (San Diego, USA). Recombinant murine macrophage colony-stimulating factor (M-CSF), interferon-gamma (IFN $\gamma$ ), and interleukin-4 (IL-4) were purchased from Peprotech (Rocky Hill, USA). The qPCR SYBR $^{\circledR}$ Green Master Mix and cDNA synthesis SuperMix kits were from Yeasen Biotech (Shanghai, China). The primers of IL-1 $\beta$, IL-6, TNF- $\alpha$, IL-12, IL-10, CD206, Arg-1, Chil3, TGF- $\beta$, CD163, CD86, iNOS, CCR7, and GAPDH were provided by Sango Biotech (Shanghai, China). Murine TNF- $\alpha$, IL-1 $\beta$, IL-6, IL-12, and IL-10 ELISA kits were from Lianke Biotech (Hangzhou, China). BCA protein assay kit, horseradish peroxidase (HRP)conjugated goat anti-rabbit/mouse lgG secondary antibody, and crystal violet were from Beyotime (Shanghai, China). Coumarin 6 and were from J\&K (Beijing, China). PC-98 T, cholesterol, and DSPE-PEG ${ }_{2000}$ were obtained from AVT (Shanghai, China). Artificial saliva (ISO/ TR10271, pH 6.8-7) was purchased from Yuanye Biotech (Shanghai, China).

The bone marrow-derived macrophages (BMDM) were obtained from bone marrow-derived monocytes from Balb/c mice according to a standard protocol [17]. Murine macrophage cells (RAW264.7), mouse fibroblast cells (L929), and human vascular endothelial cells (HUVEC) were cultured in DMEM supplemented with $1 \%$ penicillin-streptomycin and $10 \%$ FBS in $5 \% \mathrm{CO}_{2}$ at $37{ }^{\circ} \mathrm{C}$. The M1 $\Phi$ differentiation was induced using LPS $(100 \mathrm{ng} / \mathrm{mL})$ and IFN- $\gamma(20 \mathrm{ng} / \mathrm{mL})$, and M2Ф was induced using IL-4 (40 ng/mL). Streptococcus mutans (S. mutans) UA159, kindly provided by the Oral Microbiota and Systemic Disease Laboratory (Shanghai, China), were cultured anaerobically in the brain heart infusion broth (BHIB; BD, Franklin Lakes, USA) at $37^{\circ} \mathrm{C}$.

The BALB/c female mice (7-8 weeks old) were housed in an SPF facility. The animal experiments were performed according to the protocol (IACUC: 2020-06HYZ-86) approved by the Ethics Committee of Shanghai Institute of Materia Medica, Chinese Academy of Sciences.

\section{Preparation of Lipo-RSV}

The liposomes were prepared using a standard thinfilm hydration method [18]. In brief, PT-98T, cholesterol, and DSPE-PEG 2000 (20: 2: 1, w/w) were dissolved in chloroform and resveratrol in methanol. Resveratrol and phospholipids were mixed at a mass ratio of 1: 20 and the final ratio of the mixture solutions was chloroform: methanol=20: $1(\mathrm{v} / \mathrm{v})$. The organic solvent was removed using rotary evaporation for $1 \mathrm{~h}$ at
$40{ }^{\circ} \mathrm{C}$. The thus-formed thin film was hydrated using PBS (pH 7) for $30 \mathrm{~min}$ at room temperature, followed by probe sonication for $5 \mathrm{~min}$ at $10 \mathrm{~W}$. The thus-formed liposomes were extruded 21 times through polycarbonate membranes with a $200-\mathrm{nm}$ pore size by a hand held extruder (Avestin, Ottawa, Canada) and then purified using a Sephadex G-50 column (GE Healthcare, Boston, MA, USA) to remove free resveratrol.

The prepared liposomes were mixed with Pluronic ${ }^{\circledR}$ F127 $\left(20 \% \mathrm{w} / \mathrm{w}, 20^{\circ} \mathrm{C}\right)$ to obtain a homogeneous liposomal dispersion before the animal experiment.

\section{Characterization of liposomes}

The morphology of the Lipo-RSV was characterized by a cryo-electron microscope (Cryo-EM, FEI Talos Arctica G2, Thermo Fisher, Waltham, USA) operated at $200 \mathrm{kV}$. The zeta potential and particle size of the LipoRSV were measured by Zetasizer (Nano-ZS90, Malvern Instruments, UK).

The size, PDI, and zeta potential of Lipo-RSV were monitored at $37{ }^{\circ} \mathrm{C}$ and $4{ }^{\circ} \mathrm{C}$ in artificial saliva. The measurement procedure was described as in our previous report [18].

The drug-loading capacity (DL\%) and encapsulation efficiency (EE\%) of Lipo-RSV were determined by HPLC (1260 Infinity, Agilent technologies, USA) after purification by a Sephadex G100 column (GE Healthcare, Chicago, USA). The chromatographic conditions were described as follows: Agilent-C18 column $(250 \times 4.6 \mathrm{~mm}, 5 \mu \mathrm{m})$;mobile phase, $0.1 \%$ phosphoric acid in water/acetonitrile (60:40,v/v); flow rate of $1 \mathrm{ml} /$ min; detection wavelength, $375 \mathrm{~nm}$.

Lipo-RSV was dialyzed (MWCO $14 \mathrm{~K}$ ) against $50 \mathrm{ml}$ PBS ( $\mathrm{pH} 6.8$, containing $0.2 \%$ Tween-80) to maintain sink condition. It was stirred at $37{ }^{\circ} \mathrm{C}$ and a speed of $150 \mathrm{rpm}$. At the predetermined time intervals, $0.2 \mathrm{ml}$ of release medium was withdrawn and then replenished with $0.2 \mathrm{ml}$ fresh release medium. The amount of resveratrol released from Lipo-RSV was determined by HPLC.

\section{Uptake study in macrophage}

The BMDMs were seeded in the 24-well plates at a density of $5 \times 10^{4}$ cells per well and induced to the M1-like phenotype as described above. On the following day, M1 $\Phi$ were incubated with the coumarin-6-labeled liposomes $(200 \mathrm{ng} / \mathrm{ml})$ for $2 \mathrm{~h}$ and then washed three times with PBS. The cells were fixed with paraformaldehyde (4\%) for $15 \mathrm{~min}$ and stained with DAPI $(1 \mu \mathrm{g} /$ $\mathrm{ml}$ ) for $10 \mathrm{~min}$. The cells were observed by fluorescent microscopy. 


\section{In vitro cytotoxicity study}

The RAW264.7, HUVEC, or L929 were seeded into the 96-well plates at a density of $5 \times 10^{3}$ per well, respectively. After $24 \mathrm{~h}$, the cells were incubated with RSV or Lipo-RSV in different concentrations for $24 \mathrm{~h}$. Then $10 \mu \mathrm{l}$ CCK8 (diluted by fresh DMEM) was added to each well. The absorbance was detected by a microplate reader at $450 \mathrm{~nm}$. In addition, the cells were also seeded into the 24-well plates and treated with RSV $(15 \mu \mathrm{M})$ or LipoRSV $(15 \mu \mathrm{M})$. When growing to $60 \%$ confluence, the cells were then incubated with a medium containing calcein and propidium iodide for $30 \mathrm{~min}$, washed with PBS, and observed under a fluorescence microscope.

\section{Effects of Lipo-RSV on macrophage polarization and inflammatory responses}

After the BMDMs and the mouse gingival tissues were collected, RNA was extracted by adding the appropriate amount of TRIzol reagent (Tiangen, Beijing, China) according to the manufacturer's instruction, and then the reverse-transcribed cDNA was analyzed. The reverse transcription conditions were as described in a previous report [18], and the primer sequence is listed in Additional file 1: Table S1. The M $\Phi$ phenotype was detected by flow cytometry (FCM) and Western blotting, in which anti-CD206 was used to label the M2-like MФ and antiCD86 to label the M1-type MФ. Besides, the levels of cytokines secreted by the BMDM and in the gingival tissues were detected by the ELISA kits. The antibody information is listed in Additional file 1: Tables S2, S3, and S4.

\section{ROS scavenging activity assay}

RSV or Lipo-RSV in a conditioned medium with $1 \mu \mathrm{g} / \mathrm{ml}$ of LPS was added to the BMDMs for $2 \mathrm{~h}$ incubation. The BMDMs were then incubated with DCFH-DA (Beyotime, Shanghai, China) for $30 \mathrm{~min}$, and subjected to flow cytometric measurement and fluorescence microscopy.

\section{Bacteria antibiotic susceptibility assay in vitro}

RSV and Lipo-RSV at a final concentration of $15 \mu \mathrm{M}$ were added to bacterial suspension $\left(1 \times 10^{6} \mathrm{CFU} / \mathrm{ml}\right)$ and incubated at $37^{\circ} \mathrm{C}$ for $6 \mathrm{~h}$. After diluted 1,000 times, $50 \mu \mathrm{l}$ of the bacteria suspension was applied onto agar plates and incubated at $37^{\circ} \mathrm{C}$ for $24 \mathrm{~h}$.

\section{Therapeutic efficacy in vivo}

The ligature-induced periodontitis model was developed by ligation with $5-0$ silk into the subgingival and injected with $10 \mu \mathrm{l} P . g$-LPS $(1 \mathrm{mg} / \mathrm{ml})$ for 2 weeks [19]; the model was used to estimate the therapeutic effects of Lipo-RSV. Briefly, the model animals were randomly divided into 4 groups $(\mathrm{N}=4$ /group). The groups were given $10 \mu \mathrm{lPBS}$, $4 \mu \mathrm{g}$ resveratrol, $4 \mu \mathrm{g}$ Lipo-RSV, or $2 \mu \mathrm{g}$ minocycline hydrochloride, respectively, in the gingival sulcus every other day. Meanwhile, a control group was set up using the healthy mice. After 2 weeks, the gingiva and upper jaw were harvested for histology analysis (H\&E), methylene blue staining, and immunofluorescence[8]. At the experimental endpoint, the histology examination of the major organs was performed and no pathological changes were found (Additional file 1: Figure S10).

The gingiva tissues of the mice were collected, and lysed by RIPA buffer with cocktail protease inhibitors and tissue homogenizer. The protein level of IL-1 $\beta$, IL- 6 , TNF- $\alpha$, p65, p-p65, NLRP3, COX2, Caspase1, CD206, iNOS, CD86, STAT1, STAT3, p-STAT1, and p-STAT3 in the gingiva were detected by a standard Western blotting protocol. The protein expression was analyzed by ImageJ $(\mathrm{n}=3)$. In addition, qPCR assay was also performed to detect the mRNA expression of $I L-1 \beta, I L-6, T N F-\alpha$, $C D 86, C D 163, A r g-1$, and TGF- $\beta$ in the gingiva tissues $(\mathrm{n}=3)$.

In another animal experiment, the periodontitis model mice were treated with an anti-inflammatory drug Ibuprofen $(100 \mu \mathrm{g} /$ mouse) to compare with Lipo-RSV therapy following a procedure described above.

\section{Statistical analysis}

The data are expressed as the mean \pm standard deviation. All data are analyzed by unpaired Student's T-test (between two groups) or one-way ANOVA (between multiple groups), $\mathrm{n} \geq 3$. ${ }^{*} \mathrm{P}<0.05$, ${ }^{* *} \mathrm{P}<0.01$, ${ }^{* * *} \mathrm{P}<0.001$, $* *: * \mathrm{P}<0.0001$.

\section{Results}

\section{Characterizations of Resveratrol-loaded liposome}

The liposomes were prepared using a standard thinfilm hydration method (Fig. 1A). Cryo-EM images show the morphology of Lipo-RSV in a regular shape within $200 \mathrm{~nm}$ (Fig. 1B). The mean size of the Lipo-RSV was about $136 \mathrm{~nm}$, and the zeta potential was $-11 \mathrm{mV}$ (Additional file 1: Table S5). The characterization results of Lipo-RSV in 20\% Pluronic F127 were shown in Additional file 1: Table S5 and Figure S1. Lipo-RSV was stable in $20 \%$ Pluronic F127.

The drug-loading capacity (DL\%) and encapsulation efficiency (EE\%) of Lipo-RSV were measured to be $81.3 \%$ and 3.9\% (Additional file 1: Table S6).

The Lipo-RSV remained stable in the artificial saliva at $37{ }^{\circ} \mathrm{C}$ (mimicking the oral cavity temperature) or at $4{ }^{\circ} \mathrm{C}$ (storage conditions); there were just minor changes in size (Fig. 1E, 1G, H), suggesting the stability of LipoRSV. In artificial saliva, the Lipo-RSV exhibited a sustained release effect, and around $60 \%$ of the drug was 

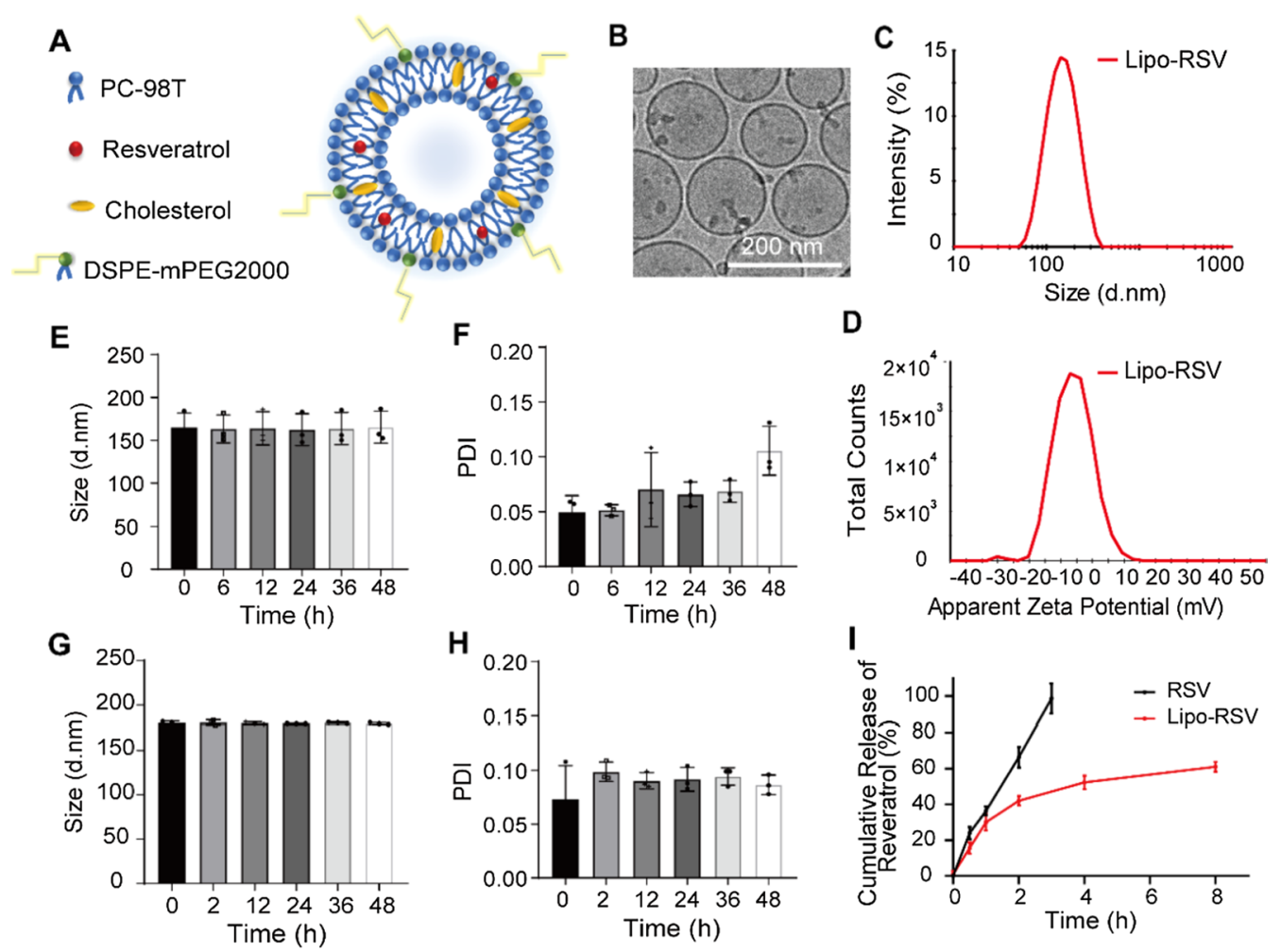

Fig. 1 Characterization of Lipo-RSV. A Schematic illustration of Lipo-RSV. B Cryo-EM of Lipo-RSV. C Size distribution of Lipo-RSV. D $\zeta$ potential measurement. E, F Stability of Lipo-RSV in artificial saliva $\left(4^{\circ} \mathrm{C}\right) . \mathbf{G}, \mathbf{H}$ Stability of Lipo-RSV in artificial saliva $\left(37^{\circ} \mathrm{C}\right) . \mathbf{I}$ In vitro release of resveratrol from Lipo-RSV

released in $8 \mathrm{~h}$ (Fig. 1I). Notably, Lipo-RSV acted in the inflammatory macrophages that are rich in the pathological site of periodontitis. Once the locally administered liposomes are taken up by the macrophages, the released drug will induce the macrophage repolarization that occurs within $24 \mathrm{~h}$ according to a routine invitro induction protocol [20].

\section{Cellular uptake and cytotoxicity studies}

After incubation with the coumarin 6-labeled liposomes, the BMDMs were observed under a fluorescence microscope and the results showed a high uptake efficiency in the BMDMs within $2 \mathrm{~h}$ (Additional file 1: Figure S2A).

At a dose of $15 \mu \mathrm{M}$, both RSV and Lipo-RSV showed little cytotoxicity in the HUVEC, RAW264.7, and L929 cells (Additional file 1: Figure S2B-D), measured by calcein/PI cell viability assay. Moreover, even when a dose was increased to $100 \mu \mathrm{M}$, all the cells retained $80 \%$ viability (Additional file 1: Figure S2E-J), determined by the CCK8 assay. It demonstrated that Lipo-RSV had good biocompatibility to these cells at the designated concentrations.

\section{Lipo- RSV drives macrophage polarization}

\section{from pro-inflammatory to anti-inflammatory phenotype}

The active M1 macrophages were treated with different concentrations of resveratrol. The qRT-PCR results showed that resveratrol up-regulated the M2-related genes (e.g., CD206 and IL-10) and downregulated the M1-related genes (e.g., iNOS and IL-1 $\beta$ ) at the concentration of $15 \mu \mathrm{M}$ (Additional file 1: Figure S3).

The qRT-PCR results revealed the re-polarization effect of Lipo-RSV. The mRNA levels of M2 macrophage markers (CD206, Arg-1, and Chil3) were up-regulated (Fig. 2D-F), and the M1 macrophage markers (CD86, iNOS, and CCR7) were down-regulated (Fig. 2G-I). Lipo-RSV inhibited the phosphorylation of STAT1 but promoted the phosphorylation of STAT3 (Fig. 2K). Therefore, it demonstrated Lipo-RSV can repolarize the macrophages from M1 to M2 phenotype through regulating p-STAT1 and p-STAT3.

After resveratrol and Lipo-RSV treatment, the morphology of macrophages showed shuttle-shaped (Additional file 1: Figure S4), which is a characteristic of M2 macrophages.

The flow cytometric analysis showed Lipo-RSV treatment increased the percentage of $\mathrm{F} 4 / 80^{+} \mathrm{CD} 206^{+}$ 


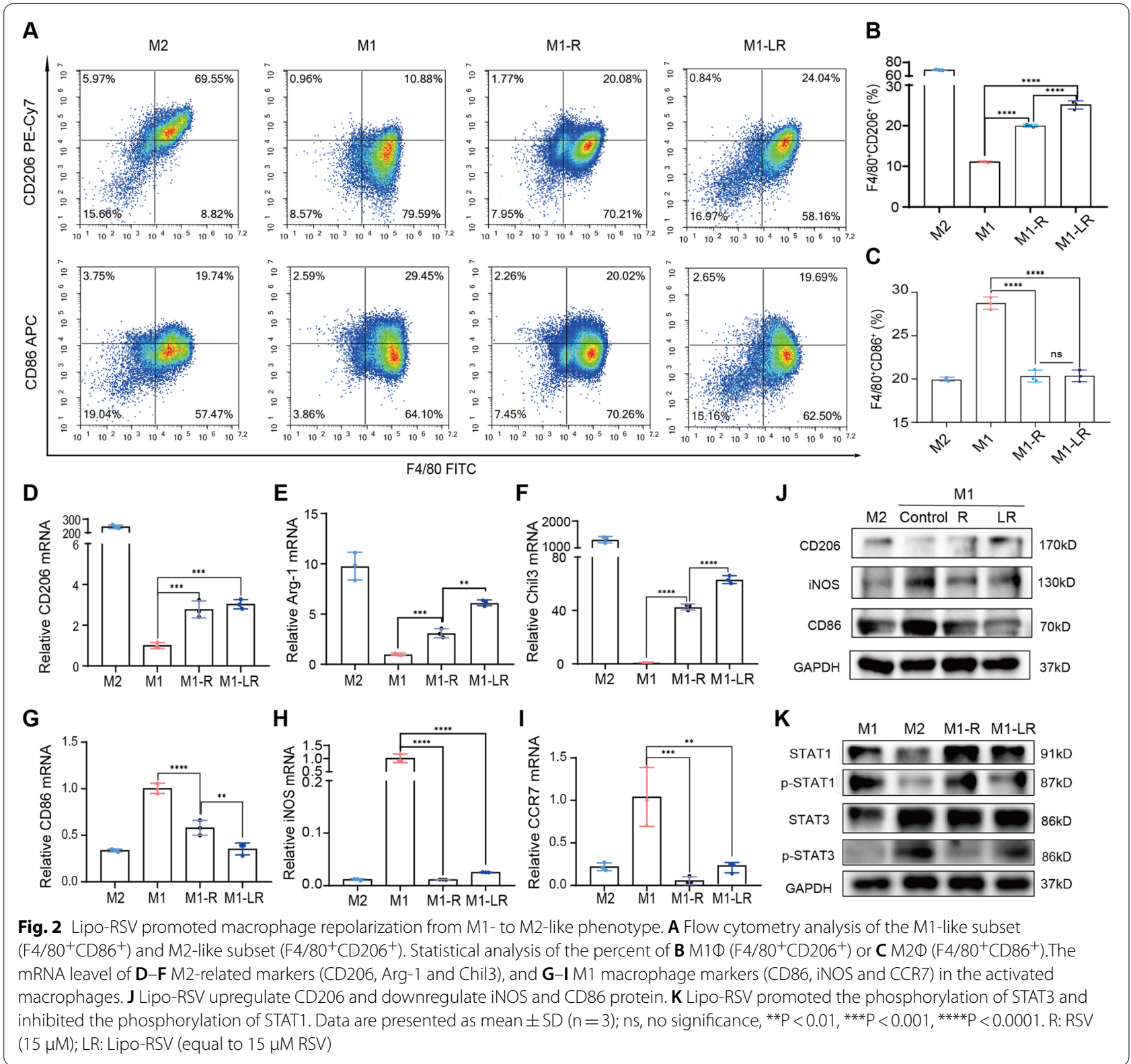

macrophages by $14 \%$ (Fig. $2 \mathrm{~B}$ ) while $\mathrm{F} 4 / 80^{+} \mathrm{CD} 86^{+}$macrophages were decreased by $8 \%$ (Fig. $2 \mathrm{C}$ ). What is more, the ratio of $\mathrm{M} 2 / \mathrm{M} 1$ was three times higher than that of untreated M1Ф (Additional file 1: Figure S5).

\section{Anti-inflammatory effect and ROS scavenging capability of Lipo-RSV in vitro}

After Lipo-RSV treatment for $24 \mathrm{~h}$, the secreted proinflammatory cytokines (IL-1 $\beta$, IL-6, TNF- $\alpha$, and IL-12) by the M1 $\Phi$ were attenuated (Fig. 3A-D). By contrast, the anti-inflammatory IL-10 was up-regulated (Fig. 3E). The transcriptional level also verified the same trend
(Fig. 3F-J). Similarly, the Western blotting results also demonstrated the decrease of the pro-inflammatory cytokines of IL-1 $\beta$, IL-6, and TNF- $\alpha$ (Fig. 3K). The effects were associated with the inhibition of the NF- $\mathrm{kB}$ signaling (Fig. 3L) and suppression of the inflammasome activation (Fig. 3M).

The ROS level in the M1Ф (stimulated by LPS, $1 \mu \mathrm{g} / \mathrm{ml}$ ) was high, but it decreased after treatment with Lipo-RSV (Fig. 4A, B). The results demonstrated that resveratrolbased treatment decreased the ROS level in the M1 $\Phi$, and Lipo-RSV exhibited a better effect than free resveratrol (Fig. 4C). 
A

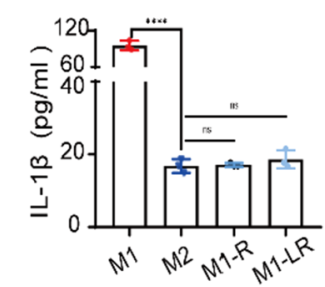

$\mathbf{F}$

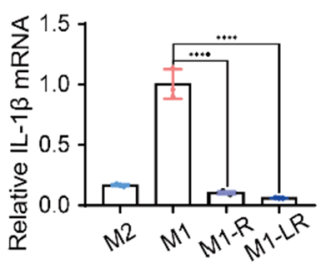

K

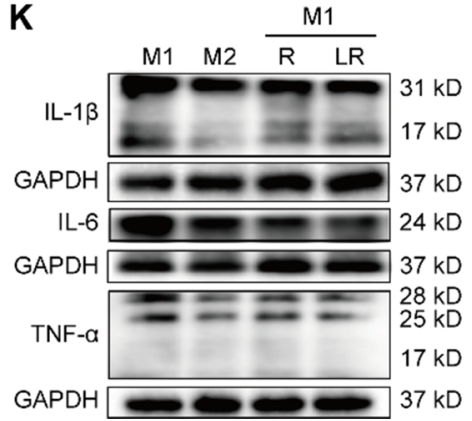

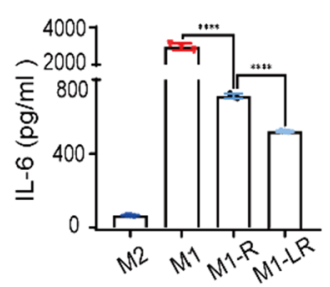

G

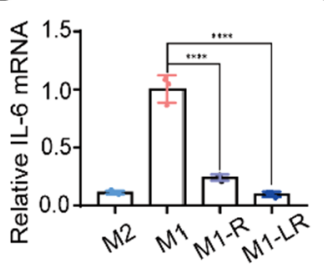

C

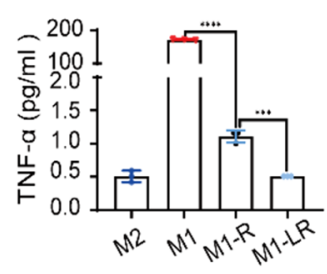

H

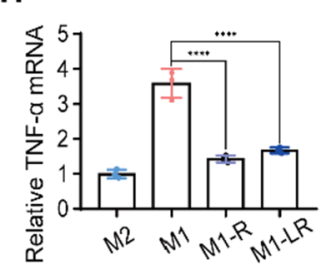

D

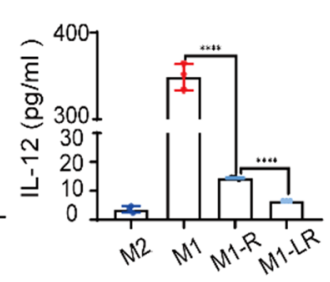

E

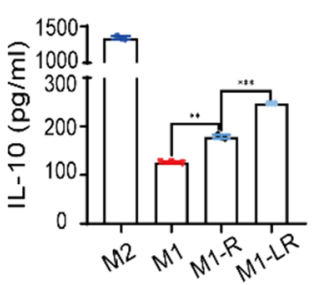

I

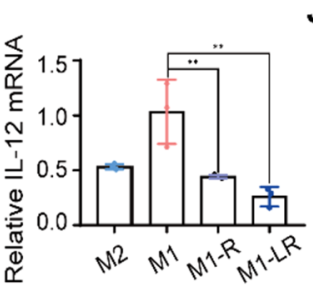

J

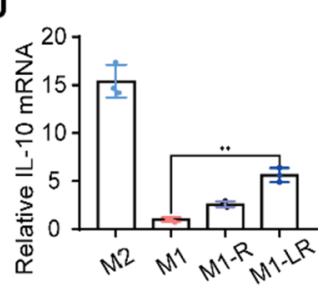

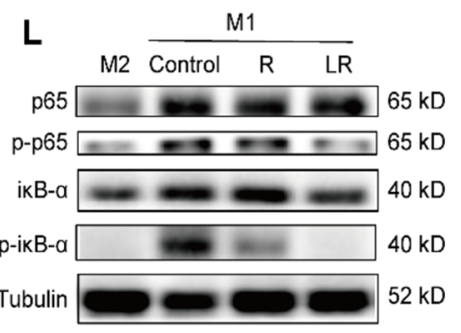

Fig. 3 Anti-inflammatory effect of Lipo-RSV in vitro. A-E ELISA analysis of the levels of IL-1 $\beta$, IL-6, TNF-a, IL-12, and IL-10. F-J mRNA levels of $I L-1 \beta, I L-6, T N F-\alpha, I L-12$, and IL-10 by qRT-PCR. K The down-regulation of IL-1 $\beta$, IL-6, and TNF-a in M1 $\Phi$ after treatment. L Expression of the NF-KB pathway-related proteins after treatment. $\mathbf{M}$ Expression of the inflammasome-related proteins after treatment. Data are presented as mean \pm SD $(n=3)$; $n$, no significance, ${ }^{* * P}<0.01,{ }^{* * *} P<0.001,{ }^{* * * *} P<0.0001$. R: RSV $(15 \mu M)$; LR: Lipo-RSV ( equal to $15 \mu M$ RSV)

A

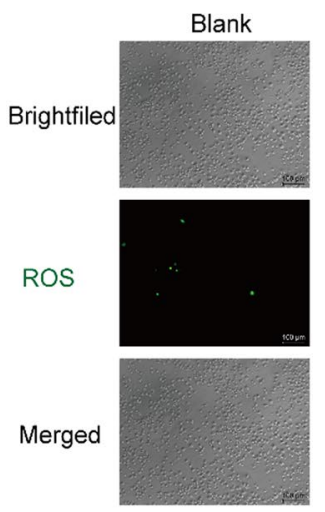

LPS
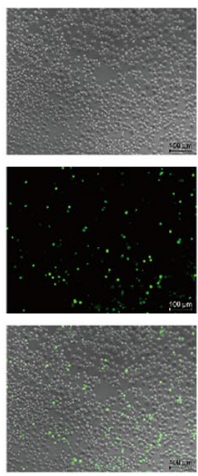
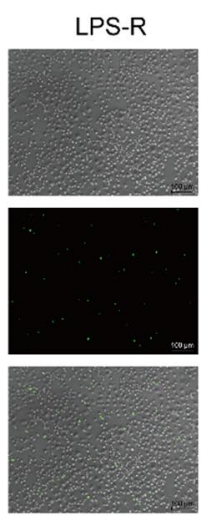
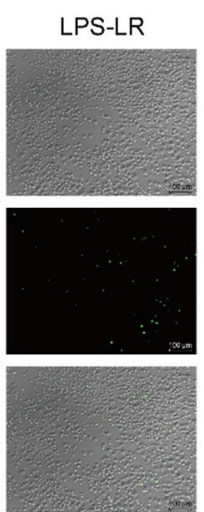

B

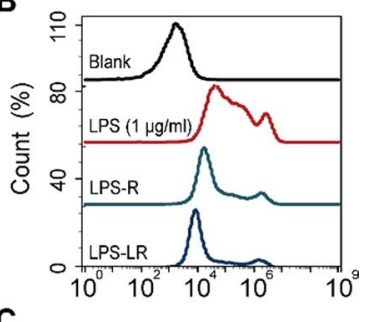

C

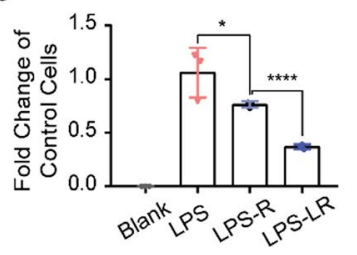

Fig. 4 ROS scavenging effect. A Fluorescence microscopy shows the clearance of ROS after treatment with Lipo-RSV. B The reduced intracellular ROS levels in M1Ф after treatment. C Statistical analysis of ROS levels. Data are presented as mean $\pm S D(n=3) ; n s, n o$ significance, ${ }^{*} P<0.05$,

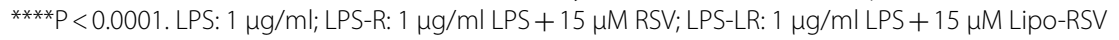




\section{Lipo-RSV ameliorates periodontitis}

An in vivo study was conducted to further confirm the effects of Lipo-RSV on periodontitis (Diagram Fig. 5A). The animal results indicated that, after two weeks of treatment, Lipo-RSV effectively ameliorated periodontitis, evidenced by a decrease in the expression of inflammatory cytokines in the gingival tissue (Fig. 5B, C) and down-regulation of the essential proteins of NF- $\mathrm{B}$ signaling (Fig. 5C) and inhibition of NLRP3 inflammatory signaling (Fig. 5D).

Furthermore, the H\&E staining showed the normalization of periodontal tissue structure after Lipo-RSV treatment (Fig. 5Ea-e). It revealed that the repair of the epithelial barrier was accompanied by the decreased number of spongiosis (indicated by the red dashed box in Fig. 5Ef-j) and the reduced inflammatory cells in the connective tissue layer (indicated by the red arrow in Fig. 5Ef-j) after Lipo-RSV treatment. The methylene blue staining of alveolar bone showed that Lipo-RSV effectively reduced the alveolar bone resorption in the lingual and buccal tissues (Fig. 5F, G).

The Western blotting result showed the increased expression of CD206 (M2Ф maker) and the decreased expression of iNOS and CD86 (M1Ф makers) in the gingiva of the periodontitis mice after treatment with LipoRSV (Fig. 6A). At the genetic level, CD86 was decreased and M2-biomarkers (CD163, Arg-1, and TGF- $\beta$ ) were increased after treatment (Additional file 1: Figure S6). The immunofluorescence results also displayed the increased expression of CD206 and reduced expression of iNOS in M1Ф $\left(\mathrm{F} 4 / 80^{+}\right)$in the gingival tissue after LipoRSV treatment (Fig. 6C). The expression of p-STAT3 was increased and p-STAT1 was suppressed in the gingival tissues from the Lipo-RSV-treated mice (Fig. 6B), with a similar trend to the in vitro results.

Antibiotic treatment (e.g., minocycline) is still the mainstream regimen in periodontitis [21], though its obvious drawbacks. However, periodontitis is a complicated disease involving multiple causes such as immune imbalance, bacterial activity, and osteogenesis. To further demonstrate the Lipo-RSV efficacy, it was also compared with an anti-inflammatory drug, ibuprofen. The results also revealed that Lipo-RSV had similar anti-bone resorption activity as ibuprofen (Additional file 1: Figure S7) and its anti-inflammatory effect was comparable to ibuprofen (Additional file 1: Figure S8).

It should be noted that RSV also has antibacterial activity [12]. Our results also revealed Lipo-RSV can efficiently inhibit Streptococcus mutans, a dental diseaserelated pathogen (Additional file 1: Figure S9). Therefore, Lipo-RSV treatment could involve multiple mechanisms.

\section{Discussion}

Minocycline is a typical antibacterial drug used in periodontitis treatment. Notably, it also has an anti-inflammatory effect [22]. The treatment efficacy of Lipo-RSV was similar to that in the minocycline-treated mice. In addition, we also compared the efficacy of Lipo-RSV with a commonly used anti-inflammatory drug ibuprofen, and the result showed similar efficacy. It implied that LipoRSV could be potentially used for treating periodontitis via immunomodulation.

Indeed, the oral cavity is a bacteria-rich environment, but normally these bacteria do not cause diseases. Only under a circumstance of host immune dysfunctions, periodontitis occurs and eventually develops into nonresolving inflammation that is no longer driven by bacterial infection. Therefore, in our study, we placed a focus on remodeling the immune microenvironment as a treatment strategy.

Macrophages play a critical role in orchestrating the immune response to infection/inflammation [23]. Clinical studies revealed that an increased ratio of M1/M2 phenotypes of macrophages positively correlates with the degree of periodontal inflammation [24]. Because of the high plasticity of macrophages, targeting macrophages has been a cutting-edge strategy for many diseases related to immune imbalance [25].

The p-STAT1 and p-STAT3 pathways are associated with the macrophage polarization between M1 and M2 phenotypes [13, 26, 27]. It has been reported that RSV has an activity of regulating macrophages through the JAK2-SATA3 pathway [13]. However, RSV is considered to be poorly druggable because of its low water solubility and stability. This limits its therapeutical application. Therefore, we developed a Lipo-RSV

(See figure on next page.)

Fig. 5 Lipo-RSV ameliorates Periodontitis. A Schematic illustration of P.g LPS-induced periodontitis in Balb/c mice and the treatment regimen. B IL-1 $\beta$ and IL-6 expression in the gingival after treatment. (a) Western blotting assay of IL-1 $\beta$ and IL-6 level in the gingiva, (b) The IL-1 $\beta$ and IL- 6 levels of the gingiva were qualified by qRT-PCR assay $(n=3)$. C p65, p-p65, and TNF-a expression in the gingival after treatment. (a) Western blotting results, (b) The TNF-a levels of the gingiva were qualified by qRT-PCR assay $(n=3)$. D NLRP3, COX2, and caspase 1 expression in the gingival after treatment. E HE staining of the gingiva. Red dashed boxes indicate epithelial junctions, and red arrows indicate infiltrating inflammatory cells. R: dental root; AB: alveolar bone. $\mathbf{F}$ Representative figures as indicated by alveolar bone loss and root exposure examined by methylene blue staining. a MH: minocycline hydrochloride, b PD: periodontitis, $\mathbf{c}$ N: normal, $\mathbf{d}$ R: RSV, e LR: Lipo-RSV. G Statistical analysis of the alveolar bone resorption length. Data are presented as mean $\pm S D(n=3) ; n s$, no significance, ${ }^{*} P<0.05,{ }^{* *} P<0.01,{ }^{* *} P<0.001,{ }^{* * *} P<0.0001$ 


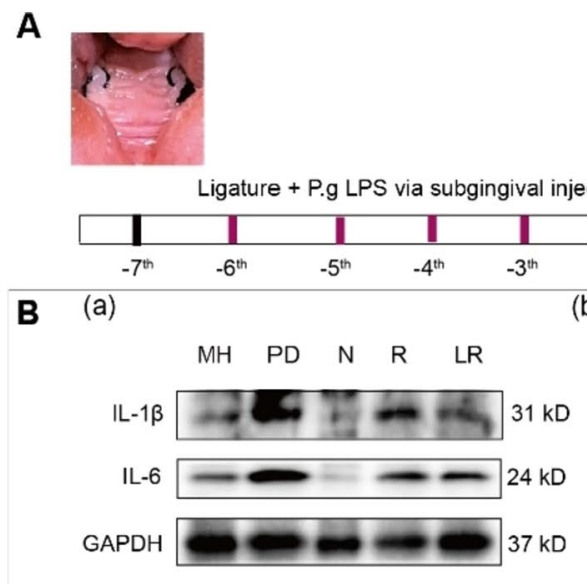

C (a)

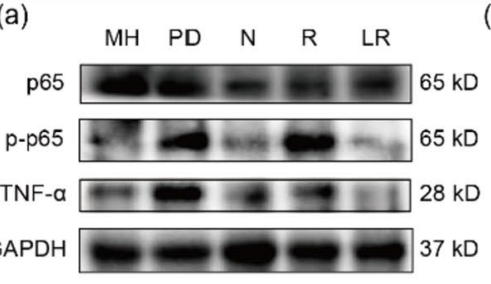

(b)

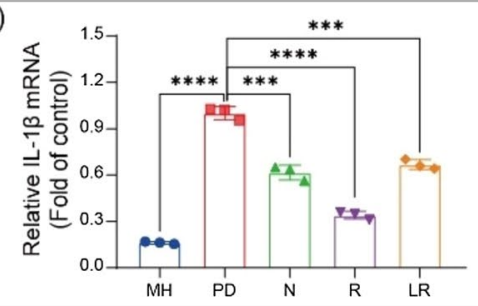

(b)

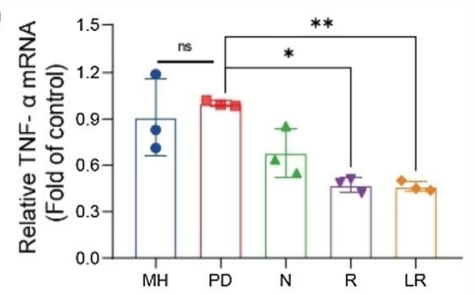

Treatment with subgingival injectio (Qod, 2 w)

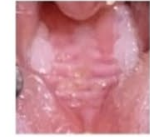

(c)

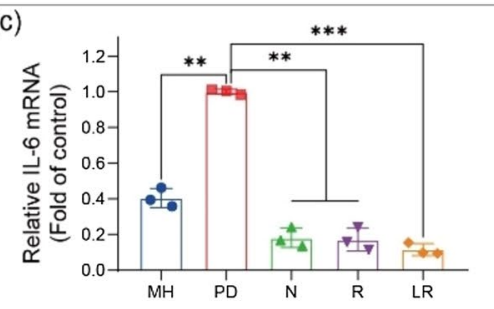

E

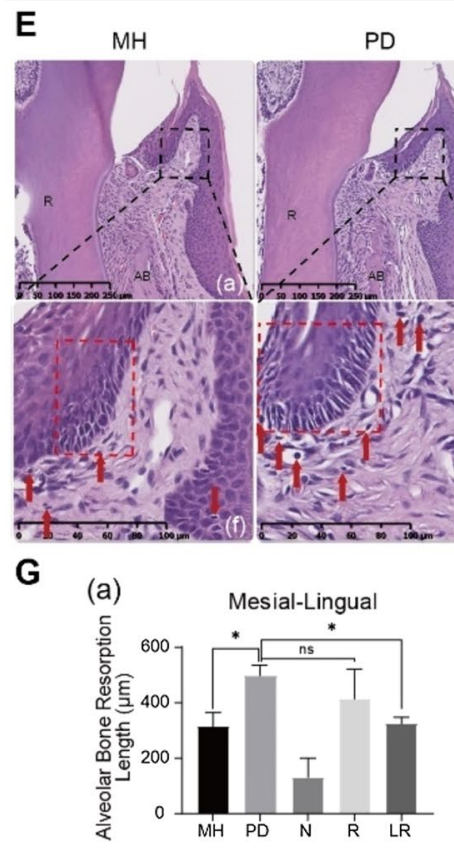

(d)

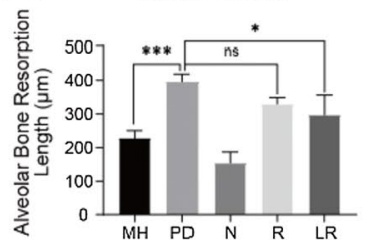

(b)

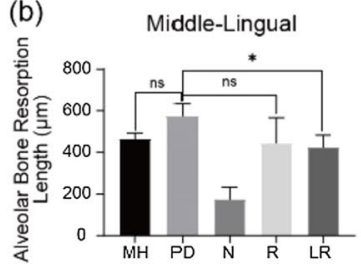

(e)

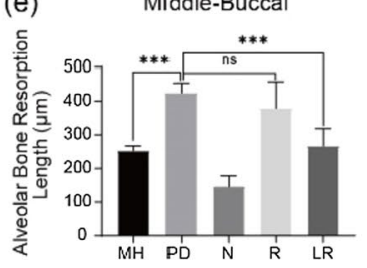

D

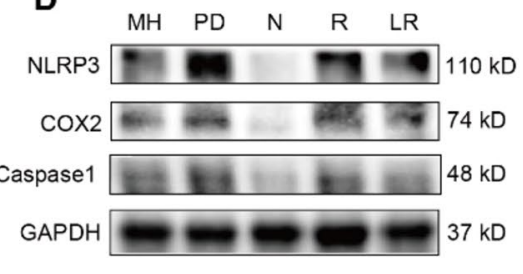

F
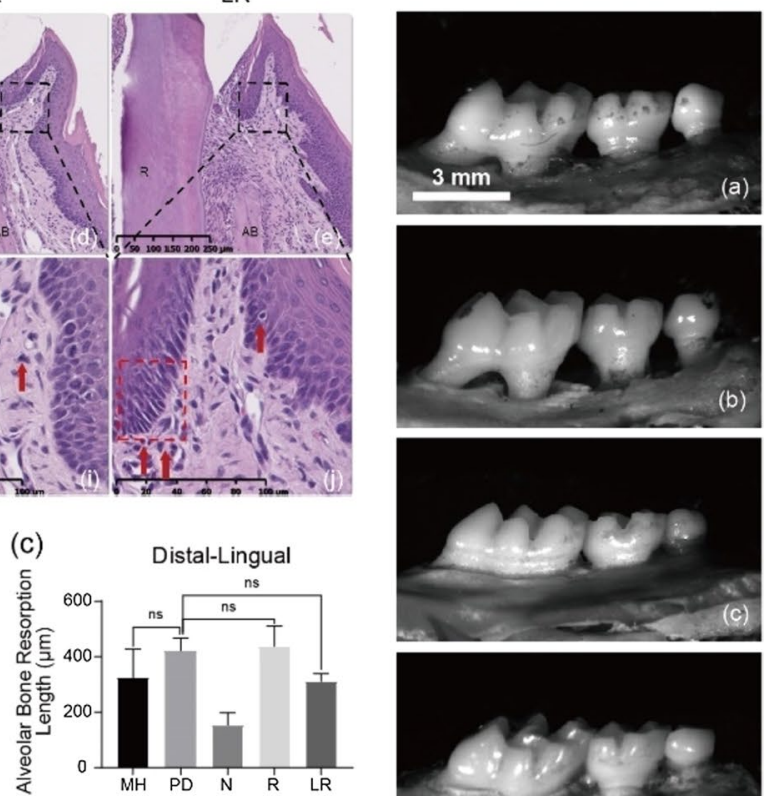

(f) Distal-Buccal
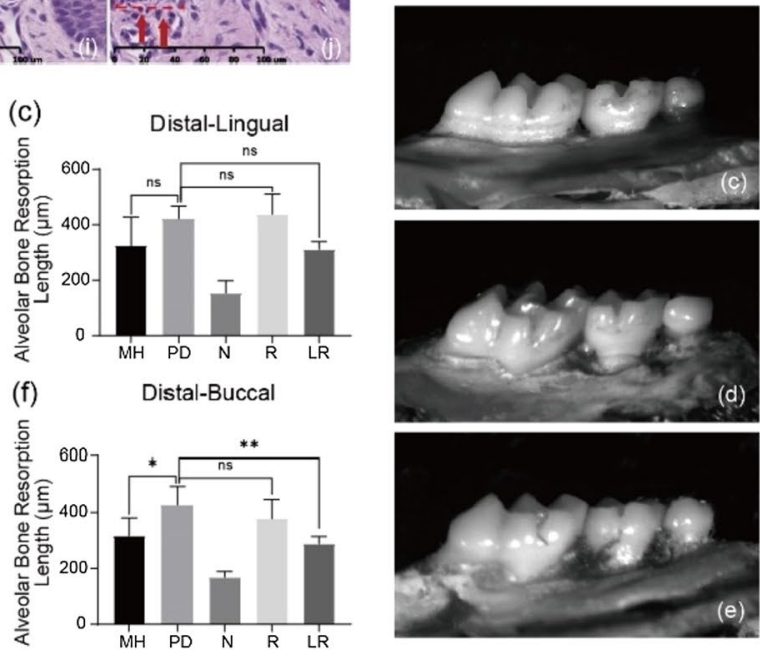

Fig. 5 (See legend on previous page.) 
A
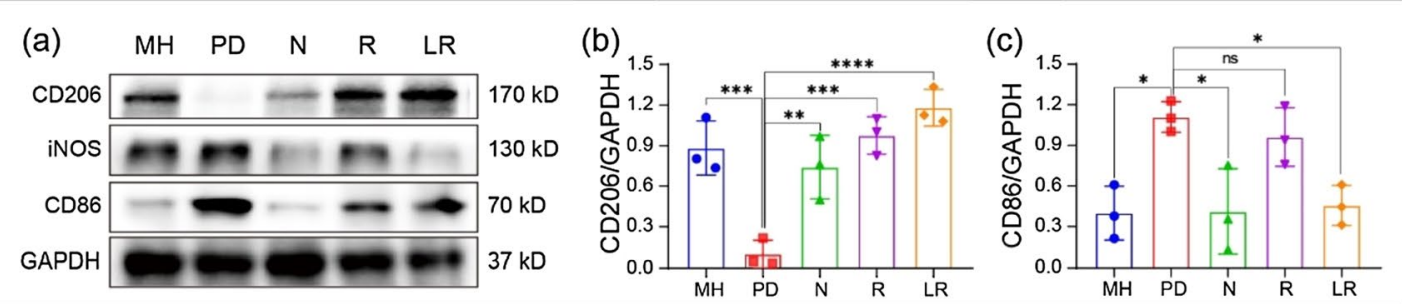

B

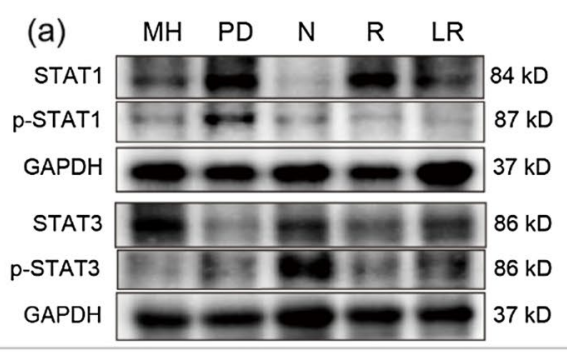

(b)

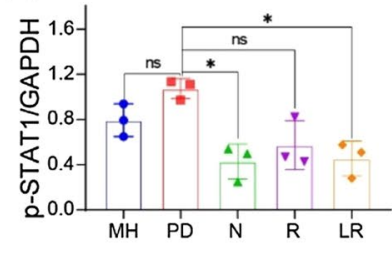

(c)

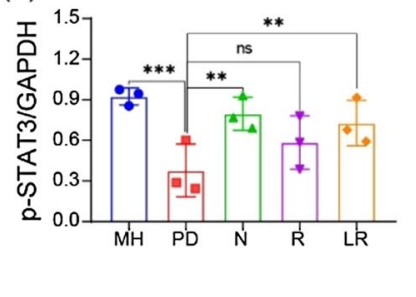

C

$\mathrm{MH}$

PD
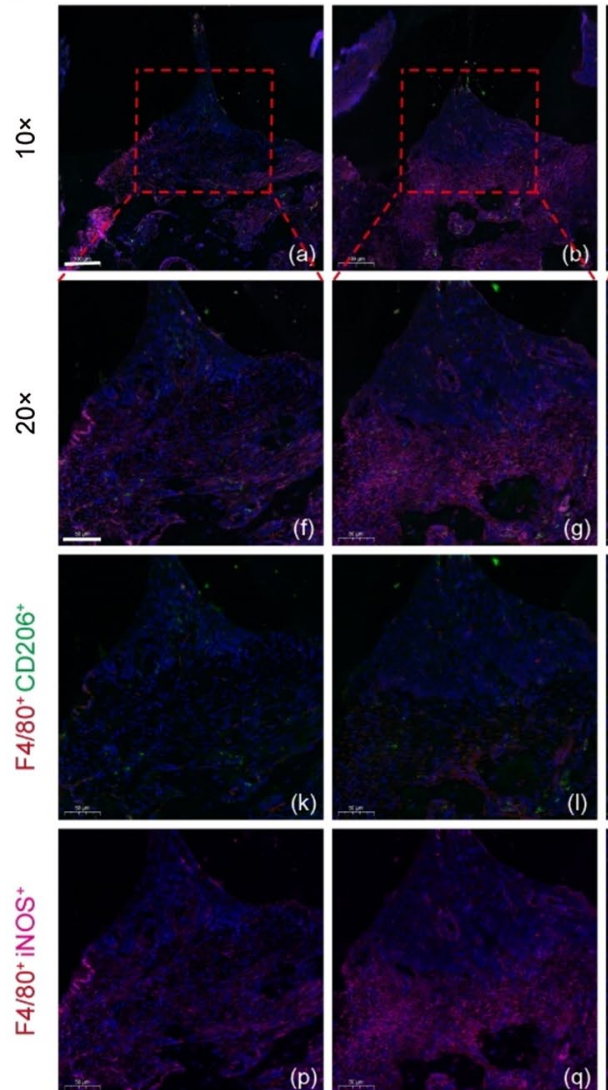

N
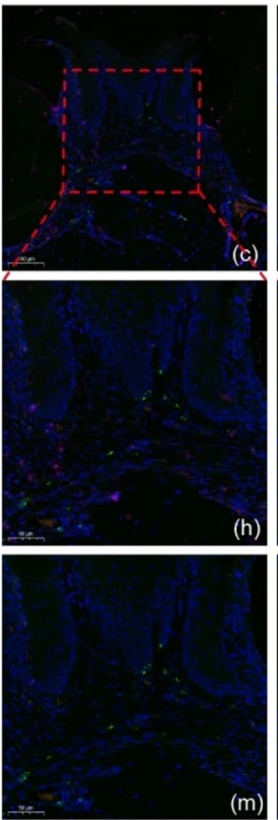

(m)

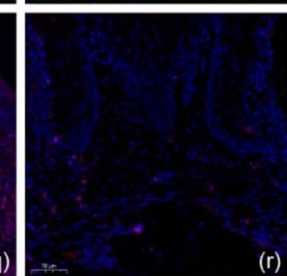

RSV
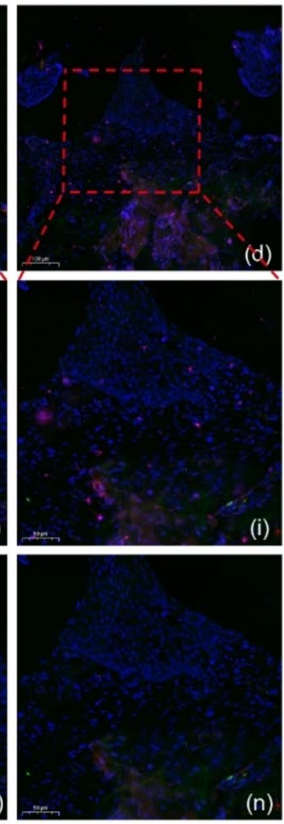

Lipo-RSV
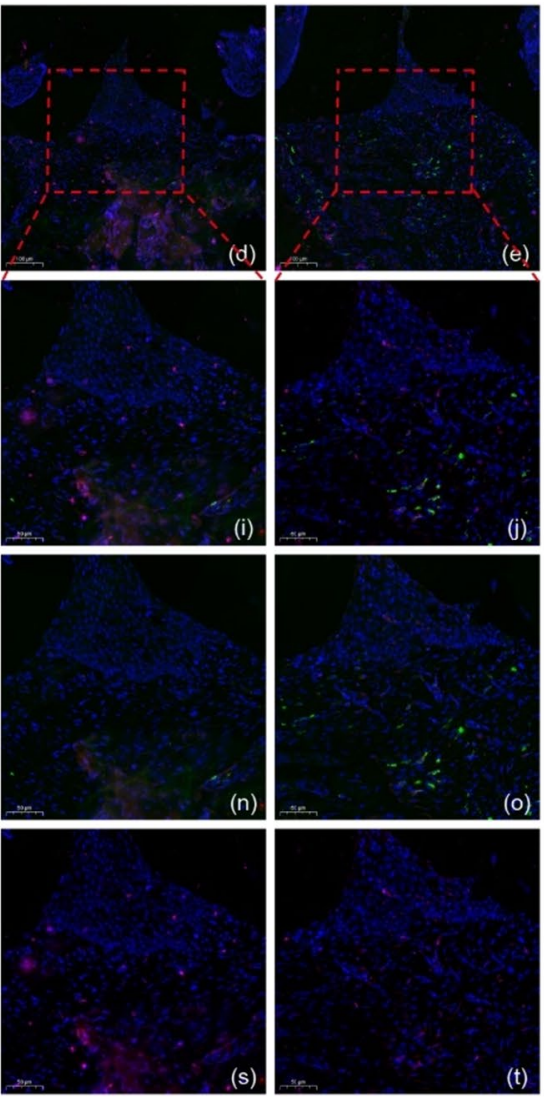

Fig. 6 Identification of macrophages in periodontitis-associated soft tissue lesions. A (a) Western blotting results of CD206, iNOS, and CD86 expression in the gingival tissues. (b, c) The CD206 and CD86 levels in gingiva were qualified analyzed by WB and ImageJ $(n=3)$. B (a) Western blotting results of the level of p-STAT1 and p-STAT3. $(b, c)$ The p-STAT1 and p-STAT3 levels in gingiva were qualified analyzed by WB and Image J $(n=3)$. C Macrophages stained for F4/80 (Cy3), CD206 (AF488), iNOS (Cy5), and cell nuclear (DAPI). Scale bar $10 \mu m(a-e)$, scale bar $50 \mu m(f-t)$. R: RSV, LR: Lipo-RSV. Data are presented as mean $\pm S D(n=3)$; $n$, no significance, ${ }^{*} P<0.05$, ${ }^{* *} P<0.01$, *** $P<0.001$, **** $P<0.0001$ 
delivery system for reprogramming macrophages from M1 to M2 to serve as an antibiotic-free strategy for treating periodontitis.

ROS is a nexus of cellular homeostasis [28, 29]. Increasing evidence showed that ROS-regulated therapy is useful for modulating the immune microenvironment [30, 31]. RSV is a potent ROS scavenger that protects against lipid peroxidation in cell membranes and DNA damage and thus promotes wound healing [32, 33]. Notably, Lipo-RSV could eliminate about $50 \%$ of ROS in M1 macrophages, and decrease the expression of NLRP3, caspase1, and IL-1 $\beta$. The excessive ROS production by inflammatory macrophages interferes with cell cycle progression and causes irreversible damage to periodontal tissues [13, 34]. ROS also plays an essential role in maintaining the inflammatory phenotype of macrophages via activating NLRP3 inflammasome and promoting IL- $1 \beta$ induction $[8,35,36]$. Therefore, ROS clearance could be an important mechanism for Lipo-RSV for remodeling the immune microenvironment. It is not fully explicated the molecular mechanisms of ROS regulating the inflammatory signaling; it is likely related to the mitochondrial state $[37,38]$.

Nanomedicine has been widely explored for its targeting delivery functions and to improve the treatment outcomes [39-41]. In addition, nanomedicine in immunoregulation has attracted great attention [42], and local administration of nanomedicine has been demonstrated for the therapeutic benefits of direct access of drugs to the targeted tissue $[7,43,44]$. The locally administered Lipo-RSV was developed for treating periodontitis via remodeling the immune microenvironment. The liposomal formulation can enhance the solubility and stability of RSV. Due to the phagocytosis nature of the inflammatory macrophages, Lipo-RSV could be beneficial for targeting macrophages. As evidence, our results showed that Lipo-RSV had superior effects on macrophage repolarization than free RSV.

\section{Conclusion}

In summary, we developed a Lipo-RSV system to treat periodontitis by modulating p-STAT1 and p-STAT3 to reprogram the macrophages from M1- to M2-like phenotype. The treatment can effectively suppress the inflammation, evidenced by the reduced secretion of proinflammatory cytokines (IL-1 $\beta$, IL-6, TNF- $\alpha$, and IL-12) and the increased anti-inflammatory factor IL-10 via inhibiting the NF-kB/NLRP3 signaling pathways. Given these, Lipo-RSV provides a promising antibiotic-free treatment method for periodontitis management and has translation potential.

\section{Abbreviations}

RSV: Resveratrol; Lipo-RSV: Resveratrol-loaded liposomes; MH: Minocycline hydrochloride; PD: Periodontitis; M1Ф: M1-like phenotype macrophage; M2Ф: M2-like phenotype macrophage; p-STAT3: Phosphorylation of signal transducer and activator of transcription 3; p-STAT1: Phosphorylation of signal transducer and activator of transcription 1; ROS: Reactive oxygen species; NFKB: Nuclear factor kappa-B; ikB-a: Inhibitor of nuclear factor kappa-B; p-ikB-a: Phospho of IKBa; NLRP3: NOD-, LRR- and pyrin domain-containing 3; COX2: Cyclooxygenase-2; TXNIP: Thioredoxin-interacting protein; IL-1 1 : Interleukin1 beta; IL-6: Interleukin-6; IL-10: Interleukin-10; IL-12: Interleukin-12; TNF-a: Tumor necrosis factor-a; P.g: Porphyromonas gingivalis; LPS: Lipopolysaccharide; Arg-1: Arginase-1; Chil3: Chitinase-like 3; iNOS: Inducible nitric oxide synthase; CCR7: CC motif chemokine receptor 7; PC-98T: Egg yolk lecithin (PC-98T); DSPE-PEG2000: 1,2-Distearoyl-sn-glycero-3-phosphoethanolamine$N$-[(polyethylene glycol)-2000]; Cryo-EM: Cryo-electron microscope; TEM: Transmission electron microscopy.

\section{Supplementary Information}

The online version contains supplementary material available at https://doi. org/10.1186/s12951-021-01175-x.

Additional file 1. Additional Tables S1-S5 and Figures S1-S9.

\section{Acknowledgements}

We thank the Molecular Imaging Center and TEM Facility at SIMM for technical support and the National Center for Protein Science Shanghai for Cryo-EM service.

\section{Authors' contributions}

JS: Conceptualization, methodology, writing—review and editing, funding acquisition, YZ: Investigation, data curation, formal analysis, investigation, writing —original draft, visualization. XZ: Validation, supervision, formal analysis, writing — review \& editing. RC: Formal analysis. JW: Investigation. JH: Investigation. BW: Supervision, resources, project administration. HL: Supervision, resources, funding acquisition. YH: Conceptualization, supervision, resources, writing - review and editing, funding acquisition. All authors read and approved the final manuscript.

\section{Funding}

This work was financially supported by the support from the National Key Research and Development Program of China (2021YFE0103100, China), NFSC (81925035, 81771116 and 81521005), Shanghai SciTech Innovation Initiative (19431903100, 18430740800), and the Project of Biobank (NO.YBKA201906) from Shanghai Ninth People's Hospital, Shanghai Jiao Tong University School of Medicine.

Availability of data and materials

They are included in the result and method sections.

\section{Declarations}

Ethics approval and consent to participate

The animal protocols were approved by the Institutional Animal Care and Use Committee (IACUC), Shanghai Institute of Materia Medica (SIMM), Chinese Academy of Sciences.

\section{Consent for publication}

Not applicable.

\section{Competing interests}

The authors declare that they have no competing interests.

\section{Author details}

${ }^{1}$ Department of Implant Dentistry, Shanghai Ninth People's Hospital, Shanghai Jiao Tong University School of MedicineCollege of Stomatology, Shanghai Jiao Tong University; National Center for Stomatology, National Clinical Research Center for Oral Diseases; Shanghai Key Laboratory of Stomatology, 
639 Zhizaoju Road, Shanghai 200011, China. ${ }^{2}$ State Key Laboratory of Drug Research, Shanghai Institute of Materia Medica, Chinese Academy of Sciences, 501 Haike Rd, Shanghai 201203, China. ${ }^{3}$ Zhongshan Institute for Drug Discovery, SIMM, CAS, Zhongshan 528437, China. ${ }^{4}$ NMPA Key Laboratory for Quality Research and Evaluation of Pharmaceutical Excipients, Shanghai 201203, China. ${ }^{5}$ Taizhou University, School of Advanced Study, Institute of Natural Medicine and Health Product, Taizhou 318000, China.

\section{Received: 7 October 2021 Accepted: 1 December 2021} Published online: 20 December 2021

\section{References}

1. Marcenes W, Kassebaum NJ, Bernabe E, Flaxman A, Naghavi M, Lopez A, Murray CJ. Global burden of oral conditions in 1990-2010: a systematic analysis. J Dent Res. 2013;92:592-7.

2. Hajishengallis G, Chavakis T. Local and systemic mechanisms linking periodontal disease and inflammatory comorbidities. Nat Rev Immunol. 2021;21:426-40.

3. Kinane DF, Stathopoulou PG, Papapanou PN. Periodontal diseases. Nat Rev Dis Primers. 2017;3:17038.

4. Spellberg B, Guidos R, Gilbert D, Bradley J, Boucher HW, Scheld WM, Bartlett JG, Edwards J. The epidemic of antibiotic-resistant infections: a call to action for the medical community from the Infectious Diseases Society of America. Clin Infect Dis. 2008;46:155-64.

5. Jepsen K, Jepsen S. Antibiotics/antimicrobials: systemic and local administration in the therapy of mild to moderately advanced periodontitis. Periodontol. 2000;2016(71):82-112.

6. Jurdzinski KT, Potempa J, Grabiec AM. Epigenetic regulation of inflammation in periodontitis: cellular mechanisms and therapeutic potential. Clin Epigenet. 2020;12:186.

7. Ouyang J, Ji X, Zhang X, Feng C, Tang Z, Kong N, Xie A, Wang J, Sui X, Deng L, et al. In situ sprayed NIR-responsive, analgesic black phosphorus-based gel for diabetic ulcer treatment. Proc Natl Acad Sci U S A. 2020;117:28667-77.

8. Bai BB, Gu CY, Lu XH, Ge XY, Yang JL, Wang CF, Gu YC, Deng AD, Guo YH, Feng XM, Gu ZF. Polydopamine functionalized mesoporous silica as ROSsensitive drug delivery vehicles for periodontitis treatment by modulating macrophage polarization. Nano Res. 2021;14:4577-783.

9. Zhang J, Zhao Y, Hou T, Zeng H, Kalambhe D, Wang B, Shen X, Huang Y. Macrophage-based nanotherapeutic strategies in ulcerative colitis. J Control Release. 2020;320:363-80.

10. Ponzoni M, Pastorino F, Di Paolo D, Perri P, Brignole C. Targeting macrophages as a potential therapeutic intervention: impact on inflammatory diseases and cancer. Int J Mol Sci. 1953;2018:19.

11. Hasturk H, Kantarci A, Van Dyke TE. Oral inflammatory diseases and systemic inflammation: role of the macrophage. Front Immunol. 2012;3:118.

12. Vestergaard $M$, Ingmer $\mathrm{H}$. Antibacterial and antifungal properties of resveratrol. Int J Antimicrob Agents. 2019;53:716-23.

13. Liu S, Du Y, Shi K, Yang Y, Yang Z. Resveratrol improves cardiac function by promoting M2-like polarization of macrophages in mice with myocardial infarction. Am J Transl Res. 2019;11:5212-26.

14. Xiao W, Zhao L, Zou H. Resveratrol promotes macrophage M2 polarization and alleviates acute gouty arthritis in mice. Acad J Second Mil Univ. 2019;40:860-5.

15. Jhaveri A, Deshpande P, Pattni B, Torchilin $\vee$. Transferrin-targeted, resveratrol-loaded liposomes for the treatment of glioblastoma. J Control Release. 2018:277:89-101.

16. Yang Z, Gao D, Guo X, Jin L, Zheng J, Wang Y, Chen S, Zheng X, Zeng L, Guo $M$, et al. Fighting immune cold and reprogramming immunosuppressive tumor microenvironment with red blood cell membrane-camouflaged nanobullets. ACS Nano. 2020;14:17442-57.

17. Kralova J, Glatzova D, Borna S, Brdicka T. Expression of fluorescent fusion proteins in murine bone marrow-derived dendritic cells and macrophages. J Vis Exp. 2018. https://doi.org/10.3791/58081.

18. Zhao Y, Yang Y, Zhang J, Wang R, Cheng B, Kalambhe D, Wang Y, Gu Z, Chen D, Wang B, Huang Y. Lactoferrin-mediated macrophage targeting delivery and patchouli alcohol-based therapeutic strategy for inflammatory bowel diseases. Acta Pharm Sin B. 2020;10:1966-76.
19. Hiyari S, Wong RL, Yaghsezian A, Naghibi A, Tetradis S, Camargo PM, Pirih FQ. Ligature-induced peri-implantitis and periodontitis in mice. J Clin Periodontol. 2018:45:89-99.

20. Liu SX, Gustafson HH, Jackson DL, Pun SH, Trapnell C. Trajectory analysis quantifies transcriptional plasticity during macrophage polarization. Sci Rep. 2020;10:12273.

21. Yang Z, Liang X, Jiang X, Guo J, Tao Y, Wang S, Cao Y, Gui S. Development and evaluation of minocycline hydrochloride-loaded in situ cubic liquid crystal for intra-periodontal pocket administration. Molecules. 2018. https://doi.org/10.1038/s41598-020-68766-w.

22. Garrido-Mesa N, Zarzuelo A, Galvez J. Minocycline: far beyond an antibiotic. Br J Pharmacol. 2013;169:337-52.

23. Jain S, Tran TH, Amiji M. Macrophage repolarization with targeted alginate nanoparticles containing IL-10 plasmid DNA for the treatment of experimental arthritis. Biomaterials. 2015;61:162-77.

24. Yang J, Zhu Y, Duan D, Wang P, Xin Y, Bai L, Liu Y, Xu Y. Enhanced activity of macrophage M1/M2 phenotypes in periodontitis. Arch Oral Biol. 2018;96:234-42.

25. Bart VMT, Pickering RJ, Taylor PR, Ipseiz N. Macrophage reprogramming for therapy. Immunology. 2021;163:128-44.

26. Li Y, Feng L, Li G, An J, Zhang S, Li J, Liu J, Ren J, Yang L, Qi Z. Resveratrol prevents ISO-induced myocardial remodeling associated with regulating polarization of macrophages through VEGF-B/AMPK/NF-kB pathway. Int Immunopharmacol. 2020;84:106508.

27. Malyshev I, Malyshev Y. Current concept and update of the macrophage plasticity concept: intracellular mechanisms of reprogramming and $\mathrm{m} 3$ macrophage "switch" phenotype. Biomed Res Int. 2015;2015:341308.

28. Dan Dunn J, Alvarez LA, Zhang X, Soldati T. Reactive oxygen species and mitochondria: a nexus of cellular homeostasis. Redox Biol. 2015;6:472-85.

29. Forrester SJ, Kikuchi DS, Hernandes MS, Xu Q, Griendling KK. Reactive oxygen species in metabolic and inflammatory signaling. Circ Res. 2018;122:877-902.

30. Ji X, Ge L, Liu C, Tang Z, Xiao Y, Chen W, Lei Z, Gao W, Blake S, De D, et al. Capturing functional two-dimensional nanosheets from sandwich-structure vermiculite for cancer theranostics. Nat Commun. 2021;12:1124.

31. Wei D, Yu Y, Zhang X, Wang Y, Chen H, Zhao Y, Wang F, Rong G, Wang W, Kang $X$, et al. Breaking the intracellular redox balance with diselenium nanoparticles for maximizing chemotherapy efficacy on patient-derived xenograft models. ACS Nano. 2020. https://doi.org/10.1021/acsnano. 0c06190.

32. Leonard SS, Xia C, Jiang BH, Stinefelt B, Klandorf H, Harris GK, Shi X. Resveratrol scavenges reactive oxygen species and effects radical-induced cellular responses. Biochem Biophys Res Commun. 2003;309:1017-26.

33. Kaleci B, Koyuturk M. Efficacy of resveratrol in the wound healing process by reducing oxidative stress and promoting fibroblast cell proliferation and migration. Dermatol Ther. 2020;33:e14357.

34. Bao XF, Zhao JH, Sun J, Hu M, Yang XR. Polydopamine nanoparticles as efficient scavengers for reactive oxygen species in periodontal disease. ACS Nano. 2018;12:8882-92.

35. Tan HY, Wang N, Li S, Hong M, Wang X, Feng Y. The Reactive oxygen species in macrophage polarization: reflecting its dual role in progression and treatment of human diseases. Oxid Med Cell Longev. 2016:2016:2795090.

36. Jun HK, Lee SH, Lee HR, Choi BK. Integrin alpha5beta1 activates the NLRP3 inflammasome by direct interaction with a bacterial surface protein. Immunity. 2012;36:755-68.

37. Kim WY, Won M, Koo S, Zhang X, Kim JS. Mitochondrial H2Sn-mediated anti-inflammatory theranostics. Nanomicro Lett. 2021;13:168.

38. Liu C, Sun S, Feng Q, Wu G, Wu Y, Kong N, Yu Z, Yao J, Zhang X, Chen $W$, et al. Arsenene nanodots with selective killing effects and their low-dose combination with ss-elemene for cancer therapy. Adv Mater. 2021;33:e2102054

39. Wei D, Yu Y, Huang Y, Jiang Y, Zhao Y, Nie Z, Wang F, Ma W, Yu Z, Huang Y, et al. A near-infrared-II polymer with tandem fluorophores demonstrates superior biodegradability for simultaneous drug tracking and treatment efficacy feedback. ACS Nano. 2021;15:5428-38.

40. Kong N, Zhang H, Feng C, Liu C, Xiao Y, Zhang X, Mei L, Kim JS, Tao W, Ji $X$. Arsenene-mediated multiple independently targeted reactive oxygen species burst for cancer therapy. Nat Commun. 2021;12:4777.

41. Gao D, Chen T, Chen S, Ren X, Han Y, Li Y, Wang Y, Guo X, Wang H, Chen $X$, et al. Targeting hypoxic tumors with hybrid nanobullets for 
oxygen-independent synergistic photothermal and thermodynamic therapy. Nanomicro Lett. 2021;13:99.

42. Wang Y, Gao D, Liu Y, Guo X, Chen S, Zeng L, Ma J, Zhang X, Tian Z, Yang Z. Immunogenic-cell-killing and immunosuppression-inhibiting nanomedicine. Bioact Mater. 2021;6:1513-27.

43. Li H, Wang Z, Archibong E, Wu Q, Chen G, Hu Q, CiT, Chen Z, Wang J, Wen $D$, et al. Scattered seeding of CART cells in solid tumors augments anticancer efficacy. Nat Sci Rev. 2021. https://doi.org/10.1093/nsr/nwab1 72.

44. Li J, Song S, Meng J, Tan L, Liu X, Zheng Y, Li Z, Yeung KWK, Cui Z, Liang Y, et al. 2D MOF periodontitis photodynamic ion therapy. J Am Chem Soc. 2021;143:15427-39.

\section{Publisher's Note}

Springer Nature remains neutral with regard to jurisdictional claims in published maps and institutional affiliations.

- fast, convenient online submission

- thorough peer review by experienced researchers in your field

- rapid publication on acceptance

- support for research data, including large and complex data types

- gold Open Access which fosters wider collaboration and increased citations

- maximum visibility for your research: over $100 \mathrm{M}$ website views per year

At BMC, research is always in progress.

Learn more biomedcentral.com/submissions 